\title{
Amperometric sensing - Bioelectroanalysis
}

\author{
Renato Seeber • Wolfgang Schuhmann • Fabio Terzi • \\ Chiara Zanardi • Nicolas Plumere • Magdalena Gebala
}

Published online: 15 March 2013

(C) Springer-Verlag Berlin Heidelberg 2013

It seems quite timely that Analytical and Bioanalytical Chemistry (ABC) is devoting a special issue to sensing. Less obvious is that the issue is limited to electrochemical sensing, even less so to amperometric sensing, bioelectrosensing included. Actually, electroanalysis is not the most widely applied analytical technique. Optical methods definitely overwhelm the electrochemical ones in popularity; once mass spectrometry, chromatography, and the relevant hyphenated techniques, are taken into account together with the optical ones, only a restricted room is seemingly left to electrochemical methods of analysis. However, especially in the field of biosensors, this general description does not alway hold and electroanalytical techniques are presently dominating successful commercial applications of biosensors.

A big change in electroanalysis has occurred in the last 30 years, caused by the invention of modified electrodes. The modification of electrode surfaces has also led to improved anchoring of highly specific biological recognition elements, allowing unprecedented performance in electrocatalytic biosensing. Electrode systems have attained increasing complexity and a large variety of electrode modifiers have been proposed, such as synthetic and natural macromolecules, different types of nanoparticles and conducting polymers or clays.

Published in the topical collections Amperometric Sensing with guest editors Renato Seeber, Fabio Terzi, and Chiara Zanardi and Bioelectroanalysis with guest editors Nicolas Plumeré, Magdalena Gebala, and Wolfgang Schuhmann.

R. Seeber $(\triangle) \cdot F$. Terzi $\cdot$ C. Zanardi

Department of Chemical and Geological Sciences,

University of Modena and Reggio Emilia, Via G. Campi, 183,

41100 Modena, Italy

e-mail: renato.seeber@unimore.it

W. Schuhmann $(\bowtie) \cdot$ N. Plumere $\cdot$ M. Gebala

Analytische Chemie, NC04/788,

Ruhr-Universität Bochum, 44780 Bochum, Germany

e-mail: wolfgang.schuhmann@rub.de
Among the novel materials developed in the last 20 years, only a limited number have been tested and used in an electrochemical context, and an even much lower number have been considered for electroanalysis. This encourages analytical chemists to closely follow developments in the field of material chemistry, basic studies in electrochemistry and electrochemical applications to potentially transfer this knowledge to effective electroanalytical systems. Concomitantly with the development of novel electrode materials, optimization of the overall system structure has become more complex, requiring its characterization using a large number of spectroscopic, microscopic and electrochemical techniques. Consequently, interdisciplinarity has become a must for the effective development of novel modified electrodes for electroanalysis. Only combinations of analytical techniques provide sufficient insight into the in-depth functioning of modified electrodes for electroanalysis and the interplay between the different components ultimately composing the complex electrode architecture. Complete knowledge, in the widest sense, is necessary to achieve the best performance of an envisaged sensor as a whole. A lot of time has passed since the years in which 'bare' electrodes were used in molecular electrochemistry studies, which were subsequently transferred to electroanalysis. More or less complex electrode mechanisms were studied only by electrochemical techniques that allowed the electrode mechanism to be fully elucidated from a qualitative and even quantitative point of view. Such a 'simple', though rigorous approach is not possible with more complex modified electrode systems any more, owing to the enormous variety of possible structures, depending on a number of possible compositions and the conditions under which the system has been realized.

A danger lies in the tendency to separate the field into two 'different kinds' of scientists doing electroanalysis: those strictly following an 'it works!' approach describing often useful effects which cannot be given an in-depth account, owing to the too great complexity of the system, 
and those keen on finding a precise link between analytical performance and structure in order to improve the former with respect to the latter. However, because the systems investigated are often too complex to afford a reliable and complete characterization in some cases, an operative approach based on the 'simple' identification of better or worse performance achieved by not always fully characterized electrode systems is used. On the other hand, scientists dealing with simpler electrode systems often speculate on the tiny changes that are suggested by the complete characterization, paying less attention to the final goal, which is, in the end, the best analytical performance. Merging the two tendencies and relevant expertise is a goal of the electroanalytical community, exploiting the best of both strategies. This step is made easier by the fact that modern physical instrumentation and expensive techniques are easier to apply. Of course, this is adding to the level of interdisciplinarity which is mandatory for successful progress in the field of electroanalytical chemistry.

It is happening more and more frequently that outstanding researchers in material sciences or other chemical or even engineering fields are facing the necessity to integrate and develop electrochemical sensing systems. This of course implies the risk that the required level of in-depth understanding of electrochemistry as well as the proper analytical treatment of the data is underestimated. This special issue consists of two sections compiled by two groups of guest editors having a substantial overlap in covering the field of electroanalysis and bioelectroanalysis. In both sections, despite our initial intention, many contributions deal with bioanalytes using either biosensors as analytical tools or other analytical detection schemes involving electroanalytical techniques. Thus, this special issue could have been rearranged in terms of the sequence of the contributions. However, we decided to keep the two sections as they were initially anticipated, i.e. separate. We wish to thank the contributors to this special issue who submitted either original research articles or critical reviews for their cooperation, their acceptance of our invitations and the pleasant and fruitful interaction. We truly hope that this special issue in the field of electroanalysis and bioelectroanalysis demonstrates to the broad readership of $\mathrm{ABC}$ the width of topics in which electroanalysis and bioelectroanalysis are indispensable for numerous applications.

\section{Topical collection amperometric sensing}

In the first section we wish to attract the attention of electroanalysts to a variety of fields that can take advantage of amperometric sensing. Matrices such as foodstuffs and environmental systems are less convincingly considered than deserved. Furthermore, industrial controls, at the level of products and waste from almost any production line, are only occasionally taken into consideration. This happens despite the fact that these are often matrices that are hard to study with optical techniques. When potential analytes in these complex matrices are considered, it is evident that the 'old' generation of electroanalytical chemists, possessing precious expertise in molecular electrochemistry on bare electrodes, is spontaneously doubtful, since the current responses obtained are necessarily far from being in agreement with those of typical well-established electrode processes. Avoiding being drawn into the opposite trap of the 'it works!' approach, we should, however, advantageously exploit similar expertise in transferring a critical view to the new approaches in electroanalysis: we believe that novel conducting materials, coupled with ad hoc developed electrode 'cleaning' procedures and proper treatment of the signals recorded, could open the door wide to electroanalysis in industrial production, especially with respect to quality control in the widest sense. Electroanalysis in 'dirty' matrices, typical of several industrial processes, aims at reaching reliability of responses in terms of sensitivity, repeatability and reproducibility even at quite high analyte concentrations. These are within ranges opposite to the femtomolar or picomolar ones, that are often the goal of researchers, and may open a new frontier to modern electroanalysis.

\section{Topical collection bioelectroanalysis}

The second part of this special issue highlights emerging trends in bioelectroanalysis. The high selectivity inherent to biorecognition elements allows the quantification of specific analytes from complex matrices that may otherwise not be accessible via standard electroanalytical methods or may be accessible only after extensive sample pretreatment. It is thus natural that bioelectroanalysis has become a method of choice for applications where sample pretreatment is excluded, such as point-of-care analytical chemistry and on-site monitoring. This is illustrated with several examples of advanced prototypes for online monitoring of lactose, glucose or lactate in complicated matrices such as food or medical samples. Implantable or hand-held point-of-care biosensors and biofuel cells are also being developed for special applications such as the tiny sample volumes from human tears. Special attention is devoted to reagentless and microfluidics-based bioelectroanalytical devices to 
achieve maximum simplicity of use. A particular focus lies in miniaturization, involving reduction of electrode size and low-volume electrochemical cells for lab-onchip technologies that may be reliably employed by the end user. In addition, novel electrode arrays and biochips are being developed for the determination of multiple analytes from a single measurement of a sample.

Although for some analytes/samples, bioelectroanalytical strategies have already been or will soon be established, in other cases more fundamental research is still required to overcome major drawbacks which are inherent in bioelectroanalysis, such as the often poor stability of biosensing devices or specifically the biological recognition element used. The development of new materials as an immobilization matrix for biorecognition elements enables one to stabilize the latter as well as eliminate or neutralize inhibitors. Moreover, not all biosensors or bioelectroanalytical strategies are exempt from electrochemical interferences. The design of direct electron transfer pathways and novel electron transfer mediators to circumvent electrochemical interferences by shifting the working potential to an interference-free potential range remains a key research focus. Finally, the detection limit may be pushed further and further with strategies such as signal amplification based on redox cycling.

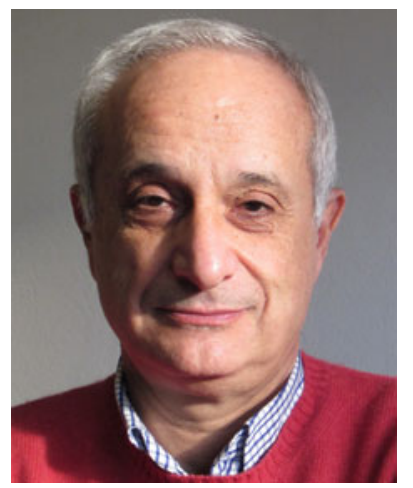

Renato Seeber has been Full Professor of Analytical Chemistry since 1986 at the universities of Sassari, Bologna and Modena, where he now works. In the past, his research interests were in the fields of molecular electrochemistry, namely electrode mechanisms of organic and inorganic compounds in aprotic media, the development of novel methods for signal filtering and for development of digital simulation techniques, thermodynamic and kinetic aspects of mobilization of iron and other metal ions in soil, and electroanalytical and chemometric methods applied to food chemistry. For almost 15 years his main interest has been in the field of modification of electrodes with intrinsically and redox conducting polymers, metal nanoparticles, graphene and relevant composites. The analytical application, even to real matrices, has always been coupled to an as deep as possible electrochemical, spectroscopic, and microscopic characterization of the electrode system, aiming at giving a rationale to its behaviour and at optimizing its structure for the best analytical performance. A parallel activity has dealt with the development and testing of electronic tongues based on amperometric sensors-differently modified electrodes -applied to characterization of food matrices.

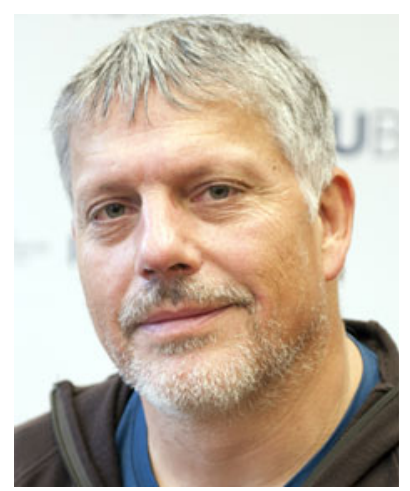

Wolfgang Schuhmann obtained his diploma in chemistry from the University of Karlsruhe, Germany, and his $\mathrm{PhD}$ degree in 1986 from the Technical University of Munich. After finishing his habilitation thesis at the Technical University of Munich in 1994, he was appointed to be a professor of analytical chemistry at Ruhr-Universität Bochum in 1996. He is the co-author of more than 360 research articles spanning topics from the development of electrochemical biosensors to microelectrochemistry, scanning electrochemical microscopy, combinatorial microelectrochemistry, localized corrosion, electrocatalysis, photoelectrocatalysis and battery materials. He is a fellow of the Royal Society of Chemistry (2005) and the International Society of Electrochemistry (2012). He has received the Biosensors \& Bioelectronics Award (2000), the Julius von Haast Fellowship Award (2008) and the Katsumi Niki Prize for Bioelectrochemistry (2012).

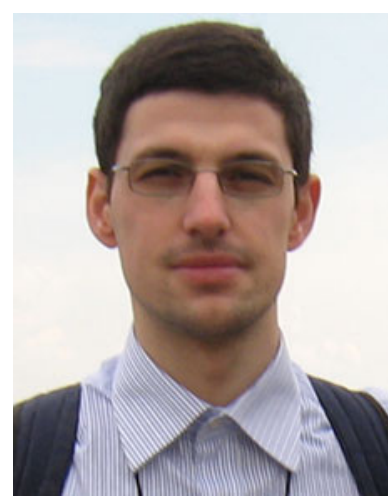

Fabio Terzi is a postdoctoral researcher in analytical chemistry at the University of Modena and Reggio Emilia. His research activities are focused on the synthesis and characterization of nanomaterials, modification of electrode surfaces using conducting polymers, self-assembled monolayers and nanomaterials, spectroscopic and microscopic characterization of electrode coatings and development and testing of amperometric sensors based on the systems realized. He has published more than 40 articles in international peer-reviewed journals.

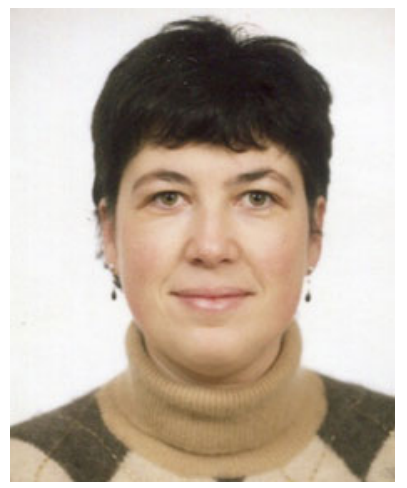

Chiara Zanardi is a researcher in analytical chemistry at the University of Modena and Reggio Emilia. Her research interests are in the field of electro analysis, particularly dealing with the development of new materials as electrode coatings possessing electrocatalytic and antifouling properties. These materials are applied in electrochemical sensors and biosensors for the quantification of meaningful analytes in food and environmental matrices.

She is the co-author of more than 50 articles in international journals and a book chapters. 


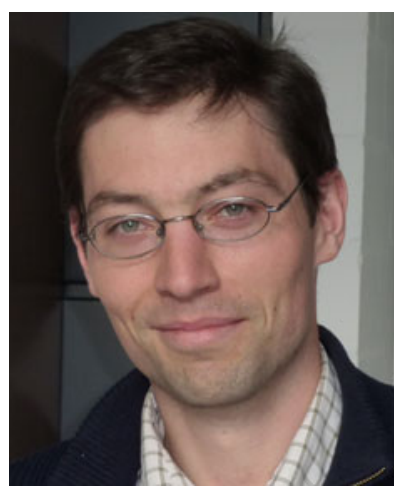

Nicolas Plumeré studied chemistry at the University of Strasbourg and the University of the West of Scotland, Glasgow, and obtained his $\mathrm{PhD}$ degree in 2009 from the University of Tübingen. He was awarded a DFG fellowship for his $\mathrm{PhD}$ work as well as a DAAD fellowship for a research visit to San Jose State University (CA, USA). After a postdoctoral stay at the laboratory of Wilbur $\mathrm{H}$. Campbell (NECi, Lake Linden, MI, USA), he started as a junior group leader at the Center for Electrochemical Sciences (CES) at RuhrUniversität Bochum in 2010 . He is the co-author of 15 publications dealing with electrochemical biosensors, single-molecule methods and redox-active nanomaterials. The research activities of his group at CES focus on the development of new strategies for efficient electrical connectivity between redox enzymes and electrodes.

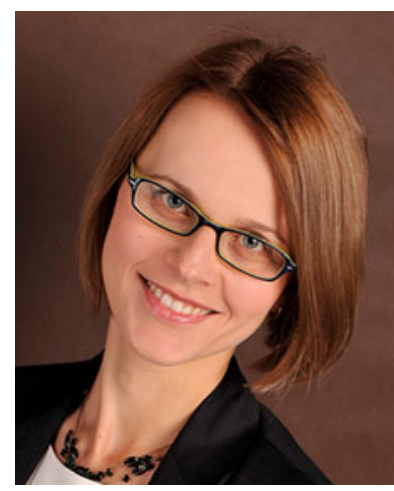

Magdalena Gebala studied biochemistry at Wroclaw University of Technology, Poland. After finishing her diploma thesis in 2006, she started her PhD work at Ruhr-Universität Bochum. In 2010, she successfully defended her thesis entitled "From impedimetric investigation of the surface architecture to labelfree DNA assays". She is the coauthor of 14 research articles. Her research interests includes the in-depth understanding of the properties of biomolecules at electrified interfaces and their impact on the design of improved biosensors and bioassays. Presently she is a postdoctoral fellow at Stanford University working on ion-RNA interactions. 